\title{
Finite Elements for Scalar Convection-Dominated Equations and Incompressible Flow Problems - a Never Ending Story?
}

\author{
Volker John • Petr Knobloch • Julia \\ Novo
}

This paper is dedicated to Ulrich Langer and Arnd Meyer on the occasions of their 65-th birthdays.

Received: date / Accepted: date

\begin{abstract}
The contents of this paper is twofold. First, important recent results concerning finite element methods for convection-dominated problems and incompressible flow problems are described that illustrate the activities in these topics. Second, a number of, in our opinion, important open problems in these fields are discussed. The exposition concentrates on $H^{1}$-conforming finite elements.
\end{abstract}

\section{Introduction}

There is a long tradition of using finite element methods for the discretization of convection-dominated scalar equations and incompressible flow problems. For convection-dominated equations, the development of the StreamlineUpwind Petrov-Galerkin (SUPG) method in 88, 41 can be considered as a major starting point of this tradition and for incompressible flow problems, the development of the theory of linear saddle point problems and the derivation of the inf-sup condition in 14,38. Since then there have been published

\footnotetext{
Volker John

Weierstrass Institute for Applied Analysis and Stochastics, Leibniz Institute in Forschungsverbund Berlin e. V. (WIAS), Mohrenstr. 39, 10117 Berlin, Germany and Freie Universität Berlin, Department of Mathematics and Computer Science, Arnimallee 6, 14195 Berlin, Germany

E-mail: john@wias-berlin.de

Petr Knobloch

Department of Numerical Mathematics, Faculty of Mathematics and Physics, Charles University, Sokolovská 83, 18675 Praha 8, Czech Republic

E-mail: knobloch@karlin.mff.cuni.cz

Julia Novo

Departamento de Matemáticas, Universidad Autónoma de Madrid, Spain.

E-mail: julia.novo@uam.es
} 
several monographs, e.g., 126, 127] about convection-dominated problems and 74, 75, 77, 112, 93. concerning incompressible flow problems. In addition, a large amount of papers has been published such that it is (nearly?) impossible to achieve an overview on the existing methods and results from numerical analysis.

Of course, in this long tradition, many fundamental ideas were developed, like different approaches for stabilization, and many basic results were proved, like a priori estimates in natural norms of the problem or the stabilized finite element scheme. During the last years, there has been the trend that the results became more special. Along with this trend, our personal observation is that the numerical analysis becomes longer and more technical. It arises the question whether this trend is the main direction for the future.

The goal of this paper is twofold. First, a number of recent results will be surveyed which show that there are still significant basic contributions to the considered topics. These results include, e.g., the finite element error analysis of algebraic stabilizations, the derivation of the EMAC form of the nonlinear term of the Navier-Stokes equations, the derivation of pressure-robust discretizations, and the progress of using and analyzing weakly divergencefree finite element methods. Considerable progress has been achieved also in the construction of stabilized methods for the time-dependent Navier-Stokes equations for which error bounds can be derived where the constant does not explicitly depend on inverse powers of the viscosity. Second, open problems in the considered topics are stated, which are important in our opinion. These problems are indicated with the symbol $\boldsymbol{\sim}$. The selection of these problems inevitably reflects our own personal views and it is certainly not complete. The sequence of their presentation does not possess any correlation with our personal opinion on their importance. The discussions in this paper focus on $H^{1}$-conforming finite element methods, with a few exceptions concerning traditional non-conforming methods for incompressible flow problems. Discontinuous Galerkin methods and $H$ (div)-conforming methods will not be covered.

The paper is organized as follows. Section 2 considers scalar convectiondiffusion-reaction equations. Incompressible flow problems are discussed in Section 3, starting with the Stokes equations, followed by the stationary NavierStokes equations, and then finished with the time-dependent Navier-Stokes equations. Finally, a summary is given.

\section{Scalar Convection-Diffusion-Reaction Equations}

Let $\Omega \subset \mathbb{R}^{d}, d \in\{2,3\}$, be a domain and $T>0$ be a final time. For the finite element error analysis, it is assumed that $\Omega$ is a Lipschitz domain with polyhedral boundary.

Scalar convection-diffusion-reaction equations are given by

$$
\partial_{t} u-\varepsilon \Delta u+\boldsymbol{b} \cdot \nabla u+c u=f \quad \text { in }(0, T] \times \Omega,
$$


where $\varepsilon>0$ is a constant diffusion coefficient, $\boldsymbol{b}$ is a convective field, and $c \geq 0$ is a scalar function describing reactions. For obtaining a well-posed problem, one has to prescribe appropriate boundary conditions and an initial condition. Besides (1), also the steady-state version

$$
-\varepsilon \Delta u+\boldsymbol{b} \cdot \nabla u+c u=f \quad \text { in } \Omega,
$$

equipped with boundary conditions, is of interest.

Equations of type (1) and (2) model the behavior of scalar quantities, like temperature, concentrations etc., that are transported in a flow field with velocity $\boldsymbol{b}$ (convection), that undergo molecular transport (diffusion), and that might interact (react) with each other. If the flow is incompressible, then it is $\nabla \cdot \boldsymbol{b}=0$.

The interesting case in applications is that convection dominates diffusion: $\|\boldsymbol{b}\|_{L^{\infty}(\Omega)} \gg \varepsilon$. In this situation, a characteristic feature of solutions of (1) and $(2)$ is the appearance of layers. Layers are thin regions where the gradient of the solution is very large. Depending on the type of layer, exponential or characteristic, the thickness of the layer region is $\mathcal{O}(\varepsilon)$ or $\mathcal{O}(\sqrt{\varepsilon})$, respectively. From the practical point of view, characteristic layers are of importance. In applications, it holds generally that $\sqrt{\varepsilon} \ll h$, where $h$ denotes the mesh width, i.e., it is not possible to resolve the layers on the used grids. This issue causes the failure of standard discretizations, like the Galerkin finite element method or the central finite difference scheme, for convection-dominated convectiondiffusion-reaction equations, since such methods try to resolve all important features of the solution. This failure is expressed in the global appearance of strong spurious oscillations.

The remedy consists in using so-called stabilized discretizations. These discretizations introduce numerical diffusion. A milestone in the development of such discretizations was the proposal of the streamline-upwind PetrovGalerkin (SUPG) method in 88, 41, which introduces numerical diffusion in streamline direction. The SUPG method is still a very popular stabilization technique. It belongs to the class of residual-based stabilizations. Further stabilizations of this type were proposed thereafter. Examples of other types of stabilized methods are the continuous interior penalty (CIP) method [50] and the local projection stabilization (LPS) method, see [27] for the first application of this method to a convection-dominated equation.

Remark 1 In our opinion, an ideal discretization of a convection-dominated convection-diffusion-reaction equation should satisfy the following properties:

1. The numerical solution should possess sharp layers.

2. The numerical solution must not exhibit spurious oscillations.

3. There should be an efficient way for computing the numerical solution.

The first two properties are connected with the accuracy of the discretization and usually correlate with results from the finite element error analysis 
in sufficiently strong norms. Since the layer width is usually smaller than the mesh width, it follows from Property 1 that it is desirable that the layer of the numerical solution is not much wider than the mesh width.

Property 2 is connected also with the physical fidelity of the numerical solution. Of course, spurious oscillations are erroneous, thus they diminish the accuracy. But even more important, they represent unphysical situations, like negative concentrations. Such unphysical results are often worthless in practice. In coupled problems, where components of the solution usually enter as coefficient in equations of the system, unphysical values might even lead to a blow-up of the simulation, e.g., as reported in 99. The non-appearance of spurious oscillations is closely connected to the Discrete Maximum Principle (DMP), i.e., the preservation of the Maximum Principle (MP) in the process of the discretization. The MP is a property of the solution of the continuous problem with a physical meaning. For instance, if $c=0$ and $f=0$ (no sources), then the MP states that $u$ takes its minimal and maximal values at the boundary of the domain $\Omega$ or at the time $t=0$. This issue is important, e.g., if $u$ is a concentration. A discretization that satisfies the DMP is also called monotone.

Properties 1 and 2 are difficult to combine. For the limit case $\varepsilon=0$, the famous Godunov Theorem states that a monotone linear discretization is at most of first order 78 . A similarly rigorous statement for the case $\varepsilon>0$ does not seem to exist. But the experience shows that higher order linear methods for convection-diffusion-reaction equations are not monotone. For this reason, many nonlinear discretizations, so-called Spurious Oscillations at Layers Diminishing (SOLD) methods or shock capturing methods, were proposed, which try to reduce or remove the spurious oscillations by using stabilization terms introducing artificial diffusion that depends on the numerical solution, see, e.g., 95, 96 for a review of such methods. For some of these schemes, results from finite element analysis, like existence of a solution or convergence, are available, e.g., in [106, 46, 120, 16]. Nonlinear discretizations require an appropriate method for the solution of the corresponding nonlinear algebraic problem. This issue is closely related to Property 3.

Linear stabilized methods, like SUPG, CIP, or LPS, satisfy Properties 1 and 3. Numerical studies with SOLD methods in [13, 95, 102 showed that most of these methods do not satisfy Property 2 neither. Also adaptive grid refinement does not cure this deficiency, see [96, unless the grid becomes so fine that the layer is resolved. Nevertheless, adaptivity is an important tool to enhance the sharpness of the layers. One of the most successful first order approaches for the numerical solution of the steady-state problem (2) was a nonlinear upwind technique [122, 105, which was proved to be a monotone method. However, difficulties with respect to the convergence of the iteration for solving the nonlinear problem were reported sometimes, e.g., in [95].

As a result of numerical assessments of stabilized discretizations in [13, 95, 102, 100, so-called algebraic stabilizations came into the focus of interest. These schemes are designed to satisfy the DMP by construction and provide reasonably sharp approximations of layers. In contrast to the methods 
discussed above, which are all based on variational formulations, the idea of algebraic stabilization is to modify the algebraic system corresponding to a discrete problem (typically the Galerkin discretization) by means of solutiondependent flux corrections. Hence, the resulting schemes are again nonlinear. A drawback of these schemes is that they have been applied successfully only for lowest order finite elements, which limits the accuracy of the computed solutions. The basic philosophy of flux correction schemes was formulated already in the 1970s in [32,141]. Later, the idea was applied in the finite element context, e.g., in 11,118. In the last fifteen years, these methods have been further intensively developed in, e.g., [107,108, 109, 110,111. Here, the name Algebraic Flux Correction (AFC) schemes was also frequently used.

Despite the attractiveness of AFC schemes, there was no rigorous numerical analysis for this class of methods for a long time. First contributions to the analysis of AFC schemes for the steady-state problem (2) can be found in 17, 18, 19. These papers are concerned with the solvability of the nonlinear problems, the validity of the DMP, and the derivation of error estimates. In particular, 19] introduced the first AFC scheme for a convection-diffusionreaction equation that satisfies both the DMP and linearity preservation on general simplicial meshes.

Although the general error estimate derived in [18 is sharp, numerical results show that for particular AFC schemes better convergence rates can be obtained, at least for certain types of meshes.

- The derivation of error estimates for AFC schemes explaining the convergence rates observed in numerical experiments for particular flux corrections is open.

- An error analysis of AFC schemes for time-dependent problems is missing.

However, also for more classical discretization techniques, the error behavior is not completely understood. For example, the question of getting an optimal rate of convergence in $L^{2}(\Omega)$ for stabilized methods is still an open problem even for steady-state problems (2). In [125], the following open question is formulated (Question 2.2):

- For general shape-regular meshes of diameter $h$ and a finite element space $V^{h}$ that includes all polynomials of degree $k \geq 1$, can one construct a finite element method for (2) whose solution $u^{h} \in V^{h}$ has the optimal $L^{2}(\Omega)$ error property

$$
\left\|u-u^{h}\right\|_{L^{2}(\Omega)} \leq C h^{k+1}\|u\|_{H^{m}(\Omega)}
$$

for some $m$ ? At present no such method is known for any value of $k$.

Note that [125] discusses open problems for methods using layer-adapted meshes, which is a topic that is not addressed in the present paper.

Most of the papers concerned with error estimates for convection-dominated problems prove global bounds that depend on norms of the solution of the continuous problem over the full domain where the equations are defined. Then, these global estimates are only meaningful for globally smooth solutions. On 
the contrary, local estimates indicate that the methods are able to produce accurate approximations in regions where the solution is locally smooth. More precisely, in general, one can choose some maximal subset that excludes all layers and prove bounds in terms of norms restricted to this subset, by means of cut-off functions [127, Part III, Theorem 3.41]. Most of the local bounds existing in the literature are for steady-state problems and even in that case the results are not complete. In [49, the authors used weighted norms for a CIP method and obtain local error estimates. For the case of the SUPG method and steady-state convection-diffusion-reaction problems, a local estimate was derived for the first time in 123. In 104,124,143, 144, see also 127, a modified SUPG method that adds artificial crosswind diffusion to the classical SUPG method is analyzed for linear finite elements. In case of evolutionary problems, the results are even more scarce. The SUPG method with spacetime finite elements was studied in 123. In 62, local bounds are obtained when the SUPG method is combined with the backward Euler scheme. The arguments used in the proof lead to proposals for the stabilization parameter that depend on the length of the time step. However, numerical experiments show that local bounds seem to hold true also with a stabilization parameter depending only on the spatial mesh width and with other time integrators. Then, the question of getting local bounds with a stabilization parameter independent of the length of the time step and the extension to others than the backward Euler method remain as open problems.

- A further development of techniques for local finite element error analysis is necessary.

Often, the error in others than the natural norm of the problem or the stabilized discrete problem is of interest, in particular the $L^{\infty}(\Omega)$ norm. Pointwise error estimates were obtained in 104 for a modified SUPG method that adds artificial crosswind diffusion for the steady-state problem (2). In this paper, the authors assume that the mesh is quasi-uniform and get pointwise accuracy of almost $\mathcal{O}\left(h^{5 / 4}\right)$ for linear finite elements under local smoothness assumptions. This pointwise estimate is sharpened in 124 to $\mathcal{O}\left(h^{11 / 8}|\log h|\right)$. In [143, by orienting the mesh in the streamline direction and imposing a uniformity condition on the mesh, the order of pointwise convergence of the modified SUPG method based on linear finite elements is increased to $\mathcal{O}\left(h^{2}|\log h|\right)$. By studying a special type of meshes, it is shown in 144 that the same method may actually converge with any order between $3 / 2$ and 2 depending on properties of the meshes. All the results mentioned above only apply to the steady-state problem 2).

- Pointwise estimates for higher order conforming finite element methods are missing.

- The derivation of pointwise estimates for time-dependent problems seems to be open.

A posteriori error estimators are used for estimating the accuracy of the computed solution and for controlling an adaptive grid refinement. In the 
case of convection-dominated problems, a posteriori error estimates should be robust with respect to the ratio of diffusion and convection, i.e., the ratio of the error and its estimate should be bounded uniformly from above and below by positive constants.

Let us consider first the steady-state equation (2). More than 15 years ago, a competitive study of a number of proposed error estimators for the SUPG solution of convection-dominated convection-diffusion equations 92 came to the conclusion that none of them is robust and that the quality of the adaptively refined grids is often not satisfactory. Since then, some contributions concerning new a posteriori error estimators for the considered class of problems and types of discretization can be found in the literature. In [138, an a posteriori error bound is presented for the error in the norm $\left(\varepsilon\|\nabla v\|_{L^{2}(\Omega)}^{2}+\|v\|_{L^{2}(\Omega)}^{2}\right)^{1 / 2}$, which is not robust. An extension of the analysis of [138] led in [139] to a robust error estimator for a norm that adds to the norm of [138 a dual norm of the convective derivative. The additional term in this norm can be only approximated. Some error bounds are also proved in 129 in the one-dimensional case in a norm that includes a semi-norm of the error of order $1 / 2$. Robust a posteriori error estimators for the $L^{1}(\Omega)$ and $L^{2}(\Omega)$ norms of the error can be found in $82,83,84$. Unlike the approaches from, e.g., [138,139, the derivation of these estimators is based on the variational multiscale theory [87. It can be applied to stabilized discretizations since their error distribution is practically local 91. The essential part in the derivation consists in computing or approximating an appropriate norm of a local Green's function, see 84 for the $P_{1}$ and $Q_{1}$ finite elements in two-dimensional situations, and 83 . for higher order finite elements in one dimension. However, the $L^{1}(\Omega)$ and $L^{2}(\Omega)$ norms are comparably weak norms, i.e., spurious oscillations of the numerical solution contribute generally little to the errors in these norms. In 101, robust residual-based a posteriori error bounds are derived for the error of the SUPG finite element approximation. The error bounds are obtained in the norm typically used in the a priori analysis of this method. The derivation of the upper bound uses some hypotheses that state essentially that the interpolation error is smaller than two times the error of the SUPG method. These hypotheses cannot be proved in practice, however, since the value of the constant in the hypothesis (i.e., two) is arbitrary and the analysis holds changing (increasing if needed) that value, one can assume that the hypotheses always hold for a value of the constant that depends on the concrete example. Because of the hypotheses, the obtained results are from the mathematical point of view only slightly less general than, e.g., the results of [139]. However, from the practical point of view, the derivation of a robust error estimator for the natural norm of the SUPG method is of interest. A similar situation can be found in 44, where the derivation of an a posteriori error estimator for the natural norm of some other stabilized discretization is also based on an assumption (a saturation assumption) that most probably is not satisfied in the worst case. In 65], essentially the same error estimator as proposed in [101] is proved to be robust in a dual norm. Robust a posteriori error estimates employing a dual norm were also derived in [136] for a wide range of stabilized methods. In 67], 
an efficient and reliable error estimator is derived for SUPG approximations to steady-state equation (2), based on the reconstruction of equilibrated fluxes. Error estimators of this type provide guaranteed error bounds without further unknown constants. The robustness of the estimator is obtained again for a dual norm of the convective term. In summary, there are very recent papers on the a posteriori error estimation of steady-state problems. However, robust estimators bound the error using dual norms, which are not very useful in practice, or, as in the case of [101, they bound the error in standard norms but requiring some extra hypotheses.

With respect to evolutionary equation (1), in [59] the extension of the error estimator proposed in 101 is considered. Numerical experiments show that the estimator works well in practice. However, from the theoretical point of view, only a one-dimensional proof is included. In [52], some specific assumptions are made on the convective term that do not hold in general. Essentially the velocity field is assumed to be decomposable in a coarse scale, slowly varying in space, and a fine scale, with small amplitude that may have strong spatial variations. Some a posteriori error bounds for quantities of interest are obtained and applied to the CIP and the SUPG methods. For the related question of goal-oriented a posteriori error estimation, the recent work of 132 should be mentioned. In the introduction of this paper, a comprehensive survey of the existing references in the literature can be found. In [132, the dual weighted residual (DWR) method is combined with the SUPG method to control the error with respect to some output functional. Both the theoretical justification of the method and some interesting numerical experiments are presented.

- Robust a posteriori error estimators for evolutionary convection-diffusion equations in general situations and with reasonable assumptions are missing.

- 125, Question 7.2]: For convection-diffusion problems, using some a posteriori error estimator combined with some strategy for mesh refinement (or for changing the local polynomial degree) can one prove convergence of the computed solution in some norm, uniformly with respect to the diffusion parameter?

A comparably new approach for the discretization of partial differential equations is isogeometric analysis (IGA) 86. The basic idea of IGA consists in using as basis functions for the discrete space the same functions that are usually utilized for the parameterization of complex domains, namely non-uniform rational B-splines (NURBS). NURBS possess a higher regularity across faces of mesh cells than continuous piecewise polynomials, which are the standard finite element basis functions. This property is advocated as a potential advantage of IGA [86]. A drawback is certainly the higher complexity of implementing IGA. Meanwhile, the concept of IGA has been applied for the numerical solution of many classes of problems. However, surprisingly, there are only few studies of the potential of IGA for convection-diffusion equations, e.g., [24, 86, 103, 134]. All studies are for academic examples, none of them is for the time-dependent problem (1). Second order NURBS were applied in a time-dependent $3 \mathrm{~d}$ ap- 
plication in [25]. In [86, it is noted that the increased smoothness may lead to a better capturing of layers and the summary of 103 emphasizes the large potential of IGA, in combination with the SUPG method, for the simulation of scalar convection-diffusion equations.

- Despite these promising initial experiences there seem to be so far no serious efforts to establish IGA as an alternative to standard $H^{1}$-conforming finite elements for the solution of convection-diffusion equations. Will this situation change in future?

The ultimate open problem for the numerical solution of convection-dominated convection-diffusion-reaction equations is the following:

- Construct methods that satisfy all properties stated in Remark 1.

So far there is no such finite element method available.

\section{Incompressible Flow Models}

Flows of incompressible Newtonian fluids are modeled by the incompressible Navier-Stokes equations, given here already in dimensionless form,

$$
\begin{aligned}
\partial_{t} \boldsymbol{u}-2 \nu \nabla \cdot \mathbb{D}(\boldsymbol{u})+(\boldsymbol{u} \cdot \nabla) \boldsymbol{u}+\nabla p & =\boldsymbol{f} \text { in }(0, T] \times \Omega, \\
\nabla \cdot \boldsymbol{u} & =0 \text { in }(0, T] \times \Omega,
\end{aligned}
$$

where $\boldsymbol{u}$ is the velocity field, $p$ the pressure, $\boldsymbol{f}$ represents body forces, and the parameter $\nu$ is a dimensionless viscosity. The inverse of $\nu$ is usually called Reynolds number and the velocity deformation tensor is the symmetric part of the velocity gradient $\mathbb{D}(\boldsymbol{u})=\left(\nabla \boldsymbol{u}+\nabla \boldsymbol{u}^{T}\right) / 2$. Note that using the divergence constraint, one obtains

$$
-2 \nu \nabla \cdot \mathbb{D}(\boldsymbol{u})=-\nu \Delta \boldsymbol{u} .
$$

Problem (3) has to be equipped with appropriate boundary conditions and an initial condition for the velocity. Based on the particular flow to be studied, also a stationary version of (3) and even a linear version without convective term, the so-called Stokes equations, can be applied.

Remark 2 The incompressible Navier-Stokes equations possess the following features that might give rise to difficulties in the analysis, numerical analysis, or in simulations:

- System (3) is a coupled system of a vector field $\boldsymbol{u}$ and a scalar function $p$ of saddle point character.

- The Navier-Stokes equations are nonlinear through the convective term $(\boldsymbol{u} \cdot \nabla) \boldsymbol{u}$.

- In applications, the convective term $(\boldsymbol{u} \cdot \nabla) \boldsymbol{u}$ often dominates the viscous term $-2 \nu \nabla \cdot \mathbb{D}(\boldsymbol{u})$. If the dominance is strong, the flow becomes turbulent. 
Possible boundary conditions for (3) (on parts of the boundary of $\Omega$ ) include Dirichlet boundary conditions (essential boundary conditions), outflow or do-nothing conditions, directional do-nothing conditions, free slip conditions, slip with friction and penetration conditions, and boundary conditions on the pressure, see [93, Sect. 2.4] for a detailed description. For academic purposes, also periodic boundary conditions are used in the analysis.

- The numerical analysis with realistic boundary conditions seems to be a wide open field for all kinds of incompressible flow problems.

Often just Dirichlet conditions on $\partial \Omega$ or periodic boundary conditions are considered in the numerical analysis. The recent proposal of the directional do-nothing condition in 36] was motivated by the fact that no analytical way could be found to control possible inflow in the case of the do-nothing condition.

\subsection{The Stokes Equation}

The Stokes equations are given by

$$
\begin{aligned}
-\nu \Delta \boldsymbol{u}+\nabla p & =\boldsymbol{f} \text { in } \Omega, \\
\nabla \cdot \boldsymbol{u} & =0 \text { in } \Omega,
\end{aligned}
$$

together with appropriate boundary conditions. From the three difficulties inherent to the Navier-Stokes equations, only the coupling of $\boldsymbol{u}$ and $p$ is present. Note that (4) can be scaled with $\nu^{-1}$ such that after having defined a new pressure and a new right-hand side, one obtains an equation without parameter. However, for illustrating some issues in this section, it is of advantage to discuss form (4).

Since velocity and pressure are coupled in all incompressible flow models, the open problems stated in this section are also present for the Navier-Stokes equations.

To simplify the presentation, from now on the case of homogeneous Dirichlet boundary conditions

$$
\boldsymbol{u}=\mathbf{0} \text { on } \partial \Omega
$$

will be considered. Then, system (4) can be transformed to a weak or variational formulation: Find $(\boldsymbol{u}, p) \in V \times Q=H_{0}^{1}(\Omega)^{d} \times L_{0}^{2}(\Omega)$ such that

$$
\begin{aligned}
(\nu \nabla \boldsymbol{u}, \nabla \boldsymbol{v})-(\nabla \cdot \boldsymbol{v}, p) & =\langle\boldsymbol{f}, \boldsymbol{v}\rangle \forall \boldsymbol{v} \in V, \\
(\nabla \cdot \boldsymbol{u}, q) & =0 \quad \forall q \in Q .
\end{aligned}
$$

The well-posedness of problem (5) has been well known since the pioneering works [14,38] on the theory of linear saddle point problems. A key in this theory is the fact that $V$ and $Q$ satisfy an inf-sup condition

$$
\inf _{q \in Q \backslash\{0\}} \sup _{\boldsymbol{v} \in V \backslash\{\mathbf{0}\}} \frac{(\nabla \cdot \boldsymbol{v}, q)}{\|\nabla \boldsymbol{v}\|_{L^{2}(\Omega)}\|q\|_{L^{2}(\Omega)}} \geq \beta_{\text {is }}>0 .
$$


Let $V^{h}$ and $Q^{h}$ be finite element spaces for velocity and pressure, respectively, that are conforming, i.e., $V^{h} \subset V$ and $Q^{h} \subset Q$. The finite element formulation of these commonly called mixed methods reads as follows: Find $\left(\boldsymbol{u}^{h}, p^{h}\right) \in V^{h} \times Q^{h}$ such that

$$
\begin{aligned}
\left(\nu \nabla \boldsymbol{u}^{h}, \nabla \boldsymbol{v}^{h}\right)-\left(\nabla \cdot \boldsymbol{v}^{h}, p^{h}\right) & =\left\langle\boldsymbol{f}, \boldsymbol{v}^{h}\right\rangle \\
\left(\nabla \cdot \boldsymbol{u}^{h}, q^{h}\right) & =0 \quad \forall \boldsymbol{v}^{h} \in V^{h} \\
& \forall q^{h} \in Q^{h}
\end{aligned}
$$

Similarly to the continuous problem, the well-posedness of (6) requires the satisfaction of a discrete inf-sup condition

$$
\inf _{q^{h} \in Q^{h} \backslash\{0\}} \sup _{\boldsymbol{v}^{h} \in V^{h} \backslash\{\mathbf{0}\}} \frac{\left(\nabla \cdot \boldsymbol{v}^{h}, q^{h}\right)}{\left\|\nabla \boldsymbol{v}^{h}\right\|_{L^{2}(\Omega)}\left\|q^{h}\right\|_{L^{2}(\Omega)}} \geq \beta_{\text {is }}^{h}>0 .
$$

Usually, it is $\beta_{\text {is }}^{h} \leq \beta_{\text {is }}$, see [55] and [93, Lem. 3.53]. A notable special case with $\beta_{\text {is }}^{h}=\beta_{\text {is }}$ is the lowest order non-conforming Crouzeix-Raviart element [57], compare also [93, Thm. 3.151].

Let

$$
V_{\mathrm{div}}^{h}=\left\{\boldsymbol{v}^{h} \in V^{h}:\left(\nabla \cdot \boldsymbol{v}^{h}, q^{h}\right)=0 \forall q^{h} \in Q^{h}\right\} \subset V^{h} .
$$

The inverse of $\beta_{\text {is }}^{h}$ enters a general estimate of the best approximation error in $V_{\text {div }}^{h}$ by the best approximation error in $V^{h}$, [93, Lem. 3.60]. An alternative estimate, based on the construction of a special interpolation operator, is presented in [76]. The constant in this estimate depends on the inverse of local discrete inf-sup constants. The approach of [76] cannot be applied to the Taylor-Hood spaces $P_{2} / P_{1}$ and $Q_{2} / Q_{1}$ in three dimensions, which belong to the most popular pairs of inf-sup stable spaces.

- Clarify whether best approximation errors in $V_{\text {div }}^{h}$ can be bounded by best approximation errors in $V^{h}$ with local inf-sup constants for $P_{2} / P_{1}$ and $Q_{2} / Q_{1}$ in three dimensions.

The best approximation error in $V_{\text {div }}^{h}$ appears in the error bounds obtained in the finite element analysis. Drawing conclusions from these bounds with respect to the order of convergence, the estimate with the best approximation error in $V^{h}$ is applied and in this way, the inverse of the discrete inf-sup constant (or of local constants) enters the error bounds. Error estimates for the pressure are based on the discrete inf-sup condition (7) such that an additional factor $\left(\beta_{\text {is }}^{h}\right)^{-1}$ appears. It is important for optimal order convergence that $\beta_{\text {is }}^{h}$ is bounded uniformly away from zero. A couple of papers study whether or not $\beta_{\text {is }}^{h}$ depends on the aspect ratio, e.g., [4, 7, 8, 2, 35, 130. Most of the available results are in two dimensions.

- Further results concerning the dependency of $\beta_{\text {is }}^{h}$ or local inf-sup constants on the aspect ratio of mesh cells are needed.

The continuity equation in the weak form (5) has to be satisfied for all functions from the infinite-dimensional space $Q$, whereas in the finite element 
formulation (6), its satisfaction is required only for functions from the finitedimensional space $Q^{h}$. In the former case, one speaks of a weakly divergencefree function (one has $\nabla \cdot \boldsymbol{u}=0$ a.e. in $\Omega$ ) and in the latter case of a discretely divergence-free function. Obviously, discretely divergence-free is a weaker property and it turns out that generally the functions in $V_{\text {div }}^{h}$ are not weakly divergence-free, i.e., it is generally $\left\|\nabla \cdot \boldsymbol{u}^{h}\right\|_{L^{2}(\Omega)} \neq 0$. This property implies, from the practical point of view, that mass is not conserved. The order of convergence of $\left\|\nabla \cdot \boldsymbol{u}^{h}\right\|_{L^{2}(\Omega)}$ is generally the same as of $\left\|\nabla\left(\boldsymbol{u}-\boldsymbol{u}^{h}\right)\right\|_{L^{2}(\Omega)}$, [93. Cor. 4.24], i.e., it is not very high for popular pairs of inf-sup stable finite element spaces. An overview, discussing the topic of the violation of the mass balance for finite element discretizations can be found in 98 and a shorter introduction in [93, Sec. 4.6]. Of course, there have been attempts to construct finite element pairs with weakly divergence-free velocities, e.g., in [72, 80 . However, most of these spaces are of rather high polynomial degree and practically not used. The only exception is the class of Scott-Vogelius spaces $P_{k} / P_{k-1}^{\text {disc }}, k \geq d,[133$, for the particular case $k=d$. This class of spaces does not satisfy the discrete inf-sup condition on general grids [93, Ex. 3.73]. But it was shown in 133 . 142 that the discrete inf-sup condition is satisfied on special grids, so-called barycentric-refined grids. These grids are constructed from simplicial grids by connecting each vertex of a simplex with the barycenter of this simplex. In this way, a simplicial grid is created with possibly very small and very large angles.

- For the Scott-Vogelius pair of finite element spaces, an analysis of the impact of these angles on the discrete inf-sup constant and also on the best approximation errors in $V_{\text {div }}^{h}$ and $Q^{h}$ is not available.

Numerical studies in 131 show very promising results for the $P_{2} / P_{1}^{\text {disc }}$ pair of finite element spaces at $2 \mathrm{~d}$ examples, even for the time-dependent NavierStokes equations, such that there is some hope that the extreme angles of barycentric-refined grids do not possess a dominant impact. Moreover, these studies illustrate that $P_{2} / P_{1}^{\text {disc }}$ works well on anisotropic barycentric-refined grids. In summary, the only promising weakly divergence-free pair of standard finite element spaces is $P_{k} / P_{k-1}^{\text {disc }}, k=d$, on barycentric-refined grids.

In the context of IGA there are also proposals for constructing discrete spaces that allow the computation of weakly divergence-free velocity fields 42 , 70,71. However, for incompressible flow problems there seems to be a similar situation as described for scalar convection-diffusion equations: compared with standard finite element methods, only rather few contributions with IGA can be found in the literature in recent years, e.g., in [85,43].

In [115], a new approach has been started for improving low order mixed discretizations. A particular goal was the construction of finite element discretizations where the error bounds for the velocity do not depend on the pressure. For this reason, the new discretizations are called pressure-robust. Classical mixed methods, as the Taylor-Hood methods, are not pressure-robust. Starting point of this approach is the following observation for the continuous problem (5). Let $\boldsymbol{f} \in L^{2}(\Omega)^{d}$, then $\boldsymbol{f}$ admits a Helmholtz decomposition 


$$
\begin{gathered}
\boldsymbol{f}=P_{\text {helm }} \boldsymbol{f}+\nabla r \text { with } P_{\text {helm }} \boldsymbol{f} \in H_{\mathrm{div}}(\Omega) \text { and } \nabla r \in\left(H_{\mathrm{div}}(\Omega)\right)^{\perp} \text { where } \\
H_{\mathrm{div}}(\Omega)=\left\{\boldsymbol{v} \in L^{2}(\Omega)^{d}, \nabla \cdot \boldsymbol{v} \in L^{2}(\Omega): \nabla \cdot \boldsymbol{v}=0 \text { and } \boldsymbol{v} \cdot \boldsymbol{n}=0 \text { on } \partial \Omega\right. \\
\quad \text { in a weak sense }\} .
\end{gathered}
$$

Inserting this decomposition in the momentum balance of (5) and applying integration by parts yields

$$
-(\nu \Delta \boldsymbol{u}, \boldsymbol{v})-(\nabla \cdot \boldsymbol{v}, p)=\left(P_{\text {helm }} \boldsymbol{f}, \boldsymbol{v}\right)-(\nabla \cdot \boldsymbol{v}, r) \quad \forall \boldsymbol{v} \in V .
$$

Thus, irrotational forces $\nabla r$ are balanced by the pressure and divergence-free forces by the velocity. Or with other words, if $\boldsymbol{f}$ is changed to $\boldsymbol{f}+\nabla r$, then the solution $(\boldsymbol{u}, p)$ changes to $(\boldsymbol{u}, p+r)$. For inf-sup stable mixed finite elements that are not weakly divergence-free, this property does not hold since

$$
\left(\nabla r, \boldsymbol{v}^{h}\right)=-\left(\nabla \cdot \boldsymbol{v}^{h}, r\right)
$$

and if $r \notin Q^{h}$, this term is not completely balanced by the finite element pressure. One can also state it in such a way that classical mixed methods relax the Helmholtz decomposition. The idea of [115] was to use an appropriate reconstruction $P^{h}$ applied to the test function such that in particular $P^{h} \boldsymbol{v} \in$ $H_{\text {div }}(\Omega)$ for all $\boldsymbol{v} \in V_{\text {div }}^{h}$. The simplest situation is that $V^{h}$ is the lowest order Crouzeix-Raviart space and then the image of $P^{h}$ can be chosen to be the lowest order Raviart-Thomas space. Using now $P^{h} \boldsymbol{v}^{h}$ as test function on the right-hand side of 6 gives

$$
\left(\nabla r, P^{h} \boldsymbol{v}^{h}\right)=-\left(\nabla \cdot\left(P^{h} \boldsymbol{v}^{h}\right), r\right) .
$$

Taking $\boldsymbol{v}^{h} \in V_{\text {div }}^{h}$, which makes the pressure term on the left-hand side of (6) zero, cancels also the contribution of $r$ on the right-hand side. In this way, one can obtain velocity error bounds that do not depend on the pressure. Changing the test function introduces a consistency error that has to be analyzed. The computed finite element velocity is generally not weakly divergence-free, but a velocity approximation with this property is $P^{h} \boldsymbol{u}^{h}$. Meanwhile, the basic idea from [115] is extended to all important pairs of finite element spaces 116 113. Thus, this approach seems to be principally understood for the Stokes equations, but new issues arise for more complicated incompressible flow problems, see Section 3.3 .

Velocity error bounds for non-pressure-robust mixed methods depend on $\nu^{-1}$, e.g., compare [93, Rem. 4.33]. This dependency is sharp in the worst case, e.g., for the no-flow problem [98, Ex. 1.1]. However, in many other examples, a strong dependency of the velocity errors on $\nu^{-1}$ can be observed only on coarse grids. Then, the error reduction for small values of $\nu$ is higher than the predicted asymptotic rate of convergence until the errors become practically independent of $\nu$, compare [94] or [93, Ex. 4.34].

- It is not understood in which cases the dependency of the velocity errors on $\nu^{-1}$ is sharp and in which cases the errors become asymptotically independent of $\nu^{-1}$. 
Using inf-sup stable pairs of finite element spaces requires the implementation of different spaces for velocity and pressure as well as the solution of linear systems of saddle point type. Since these issues introduce a certain complexity, it is appealing to develop methods that use the same finite element spaces for velocity and pressure. Because such pairs of spaces do not satisfy an inf-sup condition, a stabilization becomes necessary that removes the saddle point character of the discrete problem and thus the necessity of an inf-sup condition. There are several proposals of such stabilizations, which can be grouped in two classes: stabilizations using the residual and stabilizations using only the pressure, see [93, Sec. 4.5.2] for a brief overview. A general framework for the first class is given in [31] and this class comprises the pressure-stabilized Petrov-Galerkin (PSPG) method 90,89, an absolutely stable modification of this method [31, the Galerkin least squares (GLS) method [89, and the absolutely stable method from Douglas and Wang 64. A framework for the second class is presented in 39. This class contains the Brezzi-Pitkäranta method [40, a method from Codina and Blasco [56, the LPS method [26], a method from Dohrmann and Bochev [63, and a method that penalizes the jumps of the normal derivatives of the pressure across faces [51. Numerical analysis is available for each method. However, there seems to be no guideline which ones should be preferred in practice.

- Systematic assessments of the proposed stabilized methods are missing that clarify their advantages and drawbacks and give finally proposals which ones should be preferred in simulations.

Most methods possess parameters whose optimal asymptotic choice is known. As usual in such a situation, the optimal parameter choice for a concrete problem is not clear. By construction, the violation of the mass balance is a necessary consequence of inf-sup stabilized methods, such that weakly divergencefree finite element solutions cannot be expected. However, for the $P_{1} / P_{0}$ pair and for certain types of stabilizations, a post-processing was proposed in 20 , 21,22] for computing a weakly divergence-free discrete velocity.

Using anisotropic grids is of advantage for the simulation of many flow problems. In this situation, the impact of the aspect ratio of the mesh cells on the error analysis is of interest. The literature on the dependency of the discrete inf-sup constant on the cell aspect ratio was already mentioned. Concerning the finite element error analysis for incompressible flow problems on anisotropic meshes there are only few contributions. Most of them consider infsup stabilized discretizations, via the GLS method [121, the PSPG method [6], the LPS method [33], or the Brezzi-Pitkäranta method [114]. An important goal of the analysis was the design of appropriate stabilization parameters with respect to the aspect ratio. Conforming inf-sup stable pairs are considered in $[3$ and the non-conforming Crouzeix-Raviart element is studied in 1, 10. Most of the available analysis for conforming methods is performed for $2 \mathrm{~d}$ problems. Only in [6], tensor-product grids in $3 \mathrm{~d}$ that are refined towards the boundary are considered. 
- There are only quite few results concerning the impact of the aspect ratio of anisotropic grid cells in the finite element error analysis of incompressible flow problems. Most of the available results are for two dimensions.

Error estimates for other quantities than the natural norms of the problem are important for many applications. Estimates in the $L^{\infty}(\Omega)$ norm for general finite element approximations of the Stokes problem are established on quasi-uniform meshes for domains with smooth boundary in [54]. The authors of 81] extended this result to convex polyhedral domains in three dimensions and the lowest order Taylor-Hood elements, extending also the corresponding two-dimensional result proved in [79. Further results can be found in 73 , where $L^{\infty}(\Omega)$ estimates for finite element solutions of the Stokes and the steady-state Navier-Stokes equations in convex polyhedra are obtained. In both cases, the viscosity parameter in [73] is taken to be $\nu=1$. Concerning quantities of interest, an example for the time-dependent Navier-Stokes equations shall be already mentioned here. The error of the drag and lift coefficients for inf-sup stable finite element discretizations, combined with the backward Euler scheme, is analyzed for flows around a body in [135]. Estimates are derived for errors measured in discrete versions of the norms in $L^{2}((0, t) ; \mathbb{R})$ and $L^{\infty}((0, t) ; \mathbb{R})$.

- A priori and a posteriori error analysis for quantities of interest is still a widely open topic for all kinds of incompressible flow problems.

As for convection-diffusion equations, local finite element error analysis would be of interest for incompressible flow problems.

- For incompressible flow problems, we are unaware of local bounds proved in the literature.

\subsection{The Steady-State Navier-Stokes Equations}

If $\nu$ is sufficiently large and if all data of the problem do not depend on time, then the flow field becomes stationary and it can be modeled by the steadystate Navier-Stokes equations

$$
\begin{aligned}
-\nu \Delta \boldsymbol{u}+(\boldsymbol{u} \cdot \nabla) \boldsymbol{u}+\nabla p & =\boldsymbol{f} \text { in } \Omega, \\
\nabla \cdot \boldsymbol{u} & =0 \text { in } \Omega,
\end{aligned}
$$

equipped with appropriate boundary conditions. Besides the coupling of velocity and pressure, first item in Remark 2, (8) is nonlinear, which is the second item in Remark 2. From the practical point of view, the consideration of the dominance of the convective term is not that much of importance for the steady-state situation, since uniqueness of a weak solution can be shown only in the case that the viscous term is sufficiently large compared with both, the convective term and the forces, e.g., [93. Thm. 6.20]. Otherwise, one has to consider in practice the time-dependent Navier-Stokes equations. 
For simplicity of presentation, only the case of homogeneous Dirichlet boundary conditions is discussed in the following. Then, the weak form of (8) is derived in the usual way: Find $(\boldsymbol{u}, p) \in V \times Q$ such that

$$
\begin{aligned}
(\nu \nabla \boldsymbol{u}, \nabla \boldsymbol{v})+n_{\mathrm{conv}}(\boldsymbol{u}, \boldsymbol{u}, \boldsymbol{v})-(\nabla \cdot \boldsymbol{v}, p) & =\langle\boldsymbol{f}, \boldsymbol{v}\rangle \\
(\nabla \cdot \boldsymbol{v} \in V, q) & =0 \quad \forall q \in Q,
\end{aligned}
$$

with

$$
n_{\text {conv }}(\boldsymbol{u}, \boldsymbol{v}, \boldsymbol{w})=((\boldsymbol{u} \cdot \nabla) \boldsymbol{v}, \boldsymbol{w}) .
$$

The finite element formulation with conforming methods reads as follows: Find $\left(\boldsymbol{u}^{h}, p^{h}\right) \in V^{h} \times Q^{h}$ such that

$$
\begin{aligned}
\left(\nu \nabla \boldsymbol{u}^{h}, \nabla \boldsymbol{v}^{h}\right)+n^{h}\left(\boldsymbol{u}^{h}, \boldsymbol{u}^{h}, \boldsymbol{v}^{h}\right)-\left(\nabla \cdot \boldsymbol{v}^{h}, p^{h}\right) & =\left\langle\boldsymbol{f}, \boldsymbol{v}^{h}\right\rangle \forall \boldsymbol{v}^{h} \in V^{h}, \\
\left(\nabla \cdot \boldsymbol{u}^{h}, q^{h}\right) & =0 \quad \forall q^{h} \in Q^{h}
\end{aligned}
$$

with some discrete convective term $n^{h}\left(\boldsymbol{u}^{h}, \boldsymbol{u}^{h}, \boldsymbol{v}^{h}\right)$.

An important issue is the choice of the term $n^{h}\left(\boldsymbol{u}^{h}, \boldsymbol{u}^{h}, \boldsymbol{v}^{h}\right)$. The stability analysis requires that this term is skew-symmetric, i.e., it has to vanish if the second and third argument are identical. Choosing $n^{h}(\cdot, \cdot, \cdot)=n_{\text {conv }}(\cdot, \cdot, \cdot)$, then this property is satisfied only if $\boldsymbol{u}^{h}$ is weakly divergence-free. This situation is given, e.g., for the Scott-Vogelius pairs on barycentric-refined grids. Nevertheless, the form $(9)$ is often used in simulations since it is the easiest form from the point of view of implementation. Skew-symmetric forms for arbitrary pairs of finite element spaces include

$$
\begin{aligned}
n_{\mathrm{skew}}(\boldsymbol{u}, \boldsymbol{v}, \boldsymbol{w}) & =\frac{1}{2}\left(n_{\mathrm{conv}}(\boldsymbol{u}, \boldsymbol{v}, \boldsymbol{w})-n_{\mathrm{conv}}(\boldsymbol{u}, \boldsymbol{w}, \boldsymbol{v})\right) \\
n_{\mathrm{div}}(\boldsymbol{u}, \boldsymbol{v}, \boldsymbol{w}) & =n_{\mathrm{conv}}(\boldsymbol{u}, \boldsymbol{v}, \boldsymbol{w})+\frac{1}{2}((\nabla \cdot \boldsymbol{u}) \boldsymbol{v}, \boldsymbol{w}), \\
n_{\mathrm{rot}}(\boldsymbol{u}, \boldsymbol{v}, \boldsymbol{w}) & =((\nabla \times \boldsymbol{u}) \times \boldsymbol{v}, \boldsymbol{w}) .
\end{aligned}
$$

The application of $n_{\operatorname{rot}}(\cdot, \cdot, \cdot)$ requires the use of a modified pressure, the socalled Bernoulli pressure. The forms $100-12$ are used as well in the numerical analysis as in simulations. A recent proposal from [53] is the so-called energy momentum and angular momentum conserving (EMAC) form

$$
n_{\mathrm{emac}}(\boldsymbol{u}, \boldsymbol{v}, \boldsymbol{w})=(2 \mathbb{D}(\boldsymbol{v}) \boldsymbol{u}, \boldsymbol{w})+((\nabla \cdot \boldsymbol{u}) \boldsymbol{v}, \boldsymbol{w}),
$$

which requires also a modification of the pressure. The EMAC form was derived by requiring that the discrete nonlinear term should be chosen such that the following quantities are conserved: kinetic energy (for $\nu=0, \boldsymbol{f}=\mathbf{0}$ ), momentum (for $\boldsymbol{f}$ with zero linear momentum), and angular momentum (for $\boldsymbol{f}$ with zero angular momentum). It is shown in [53] that the EMAC form is the only one that conserves all these quantities. In addition, helicity (for $\nu=0, \boldsymbol{f}=\mathbf{0}), 2 \mathrm{~d}$ enstrophy, and total vorticity are conserved by the EMAC form. Numerical studies in [53] show that the EMAC form was always among the most accurate discretizations of the convective term. Promising numerical results are also presented in 131. 
- From the theoretical point of view, the new EMAC form offers several attractive features. Further numerical studies are necessary to clarify whether this form should be preferred also in simulations.

Note that the forms $(10,, 11)$, and $(13)$ are identical to $(9)$ if the finite element velocity solution is weakly divergence-free, e.g., as given for the Scott-Vogelius pair of spaces on barycentric-refined grids. The numerical analysis might also profit from this situation since for $(9)$ only one term has to be bounded. For the time-dependent Navier-Stokes equations there are already results in this direction, compare [93, Rem. 7.40] and [131].

Using a Newton, quasi Newton, or Picard method for solving the NavierStokes equations requires the solution of a linear saddle point problem in each iteration. The efficiency of computing this solution is the key for the efficiency of the whole simulation. Recent numerical studies in 140,2 show that socalled coupled multigrid methods with Vanka-type smoothers [137] are often much more efficient than certain one-grid solvers, in particular on fine grids. However, the geometry in applications might be so complex that only one grid is available and multigrid methods cannot be applied. Among the one-grid solvers, sparse direct solvers were particularly inefficient for three-dimensional problems in [140,2]. The numerical studies in [140,2] considered as one-grid solver flexible GMRES (FGMRES) [128] with preconditioners of least squares commutator (LSC) type 68, 69. In three dimensions, this approach worked on the one hand much more efficient than the used sparse direct solver but on the other hand much less efficient than FGMRES with coupled multigrid preconditioners. An alternative one-grid approach is the augmented Lagrangian-based preconditioner proposed in [28,29].

- The construction of more efficient one-grid preconditioners for iterative solvers of linear saddle point problems arising in the discretization of the steady-state Navier-Stokes equations than currently available, in particular for problems in three dimensions with many degrees of freedom, is necessary.

Note that the situation for time-dependent problems, in particular when discretized with small time steps, is different. In this case, FGMRES with a LSCtype preconditioner that uses an iterative solver of the velocity subproblem proved to be generally much more efficient than all multigrid preconditioners in the numerical studies in [2].

Next, a posteriori error estimators for convection-dominated flow problems will be discussed. In [30], an a posteriori error estimator for a generalized Oseen problem (linearized Navier-Stokes equations) for the SUPG method is presented, leading to similar results to the ones presented in [139. A fully computable a posteriori error estimator for a generalized Oseen problem is introduced in [5], where the solution is approximated with low order conforming and conforming stabilized finite element methods, and one estimates the error in the norm $\left(\nu\|\nabla \boldsymbol{u}\|_{L^{2}(\Omega)}^{2}+c\|\boldsymbol{u}\|_{L^{2}(\Omega)}^{2}+\|p\|_{L^{2}(\Omega)}^{2}\right)^{1 / 2}$, with $c>0$ being the constant in the zeroth order term. The analysis is based on the solution of 
local Neumann-type problems that required the introduction of suitable equilibrated fluxes. The constants in the error bounds depend on inverse powers of the viscosity parameter. As it can be observed in the numerical studies of [5], the proposed estimator is not robust in the convection-dominated regime. Some a posteriori error estimators of residual-type for the Oseen problem and a stabilized scheme are presented in [23]. As in [5], the constants in the bounds depend on inverse powers of the viscosity parameter. In [45, the Navier-Stokes equations on the unit square with periodic boundary conditions are considered. Analogous assumptions to those from 52 were used for the velocity of the Navier-Stokes equations. While the assumptions of 552 do not hold in general, those in [45] are even impossible to check in practice. The constants in [45] depend exponentially on a factor proportional to the $L^{\infty}(\Omega)$ norm of the gradient of the large eddies of the exact solution. Some a posteriori error estimations are obtained for the vorticity in a weak norm.

- Deriving robust a posteriori error estimators in the convection-dominated regime is an open problem even for the Oseen equations (linearized NavierStokes equations).

Applying Newton's method for the solution of the Navier-Stokes equations or having to solve a dual linearized problem to the Navier-Stokes equations within optimization techniques or for a posteriori error estimation with the DWR method leads to linear problems of the form

$$
\begin{aligned}
-\nu \Delta \boldsymbol{u}+(\boldsymbol{w} \cdot \nabla) \boldsymbol{u}+(\boldsymbol{u} \cdot \nabla) \boldsymbol{w}+\nabla p & =\boldsymbol{g} \text { in } \Omega, \\
\nabla \cdot \boldsymbol{u} & =0 \text { in } \Omega,
\end{aligned}
$$

for given $\boldsymbol{w}$ and $\boldsymbol{g}$ satisfying $\nabla \cdot \boldsymbol{w}=0$. The zeroth order term $(\boldsymbol{u} \cdot \nabla) \boldsymbol{w}$ does not possess any sign such that coercivity of the operator corresponding to a weak form of (14) cannot be guaranteed. In particular, the available theory for the Oseen problem, which requires a zeroth order term $c u$ with $c \in L^{\infty}(\Omega), c \geq 0$, is not applicable to (14). There seems to be only little analysis for problems of type (14). In 66, a post-processing technique for increasing the accuracy of the Galerkin approximation to the Navier-Stokes equations is proposed that adds the term $c \boldsymbol{u}$ with an appropriate constant $c>0$ to the left-hand side of (14). Then, the associated bilinear form looks as follows

$$
B_{\boldsymbol{w}}(\boldsymbol{u}, \boldsymbol{v})=\nu(\nabla \boldsymbol{u}, \nabla \boldsymbol{v})+((\boldsymbol{w} \cdot \nabla) \boldsymbol{u}, \boldsymbol{v})+((\boldsymbol{u} \cdot \nabla) \boldsymbol{w}, \boldsymbol{v})+c(\boldsymbol{u}, \boldsymbol{v})
$$

for $\boldsymbol{u}, \boldsymbol{v} \in V$. For investigating coercivity of (15), the anti-symmetry of the convective term, implying $((\boldsymbol{w} \cdot \nabla) \boldsymbol{u}, \boldsymbol{u})=0$, and the estimate

$$
|((\boldsymbol{u} \cdot \nabla) \boldsymbol{w}, \boldsymbol{u})| \leq\|\nabla \boldsymbol{w}\|_{L^{\infty}(\Omega)}\|\boldsymbol{u}\|_{L^{2}(\Omega)}^{2}
$$

are used, such that

$$
B_{\boldsymbol{w}}(\boldsymbol{u}, \boldsymbol{u}) \geq \nu\|\nabla \boldsymbol{u}\|_{L^{2}(\Omega)}^{2}+\left(c-\|\nabla \boldsymbol{w}\|_{L^{\infty}(\Omega)}\right)\|\boldsymbol{u}\|_{L^{2}(\Omega)}^{2} .
$$

Choosing now $c \geq\|\nabla \boldsymbol{w}\|_{L^{\infty}(\Omega)}$ gives coercivity of $B_{\boldsymbol{w}}(\cdot, \cdot)$ with the coercivity constant $\nu$. 
- The analysis of problems of type (14) is widely open.

Note that a regularity assumption of the form $\boldsymbol{u} \in W^{1, \infty}(\Omega)^{d}$ is not unusual for the finite element analysis, in particular in the time-dependent situation, compare Section 3.3 .

\subsection{Time-Dependent Navier-Stokes Equations}

The numerical analysis of continuous-in-time discretizations and of fully discrete methods that use a fully implicit form of the nonlinear term lead to an intermediate estimate for which a Gronwall-type lemma is applied. The application of this lemma gives an exponential factor in the error bounds whose argument depends on the length of the time interval, norms of the solution, and possibly on inverse powers of the viscosity. Depending on the form of the discrete convective term, the assumptions on the smoothness of the solution, and the finite element space, different arguments are obtained.

Consider first Galerkin methods, i.e., discretizations without any stabilization terms. Using the standard skew-symmetric form of the convective term (10) and assuming $\nabla \boldsymbol{u} \in L^{4}\left(0, T ; L^{2}(\Omega)^{d \times d}\right)$, one gets the argument

$$
\frac{C}{\nu^{3}}\|\nabla \boldsymbol{u}\|_{L^{4}\left(0, t ; L^{2}(\Omega)\right)}^{4}
$$

in the exponential, compare [93, Thm. 7.35]. It was proposed in [48,12] to assume a higher regularity of the velocity, namely $\nabla \boldsymbol{u} \in L^{1}\left(0, T ; L^{\infty}(\Omega)^{d \times d}\right)$ and $\boldsymbol{u} \in L^{2}\left(0, T ; L^{\infty}(\Omega)^{d}\right)$. Then, a different technique can be applied for estimating the nonlinear term which leads to an exponential with the argument

$$
\frac{1}{2}\|\nabla \boldsymbol{u}\|_{L^{1}\left(0, T ; L^{\infty}(\Omega)\right)}+\frac{4}{\nu}\|\boldsymbol{u}\|_{L^{2}\left(0, T ; L^{\infty}(\Omega)\right)}^{2},
$$

e.g., see [93, Rem. 7.39]. Hence, the explicit dependency on the viscosity is reduced from $\nu^{-3}$ to $\nu^{-1}$. If one considers weakly divergence-free and inf-sup stable pairs of finite element spaces, like the Scott-Vogelius pair $P_{k} / P_{k-1}^{\text {disc }}$, $k \geq d$, on barycentric-refined grids or weakly divergence-free IGA methods from [42,70,71, one can use the standard form $n_{\text {conv }}(\cdot, \cdot, \cdot)$ of the convective term in the error analysis. That means, one has to bound only one term and not two terms as in other skew-symmetric formulations of the convective term. The error analysis uses the assumption $\nabla \boldsymbol{u} \in L^{1}\left(0, T ; L^{\infty}(\Omega)^{d \times d}\right)$, which guarantees the regularity of a solution of the $3 \mathrm{~d}$ incompressible Euler equations, i.e., (3) with $\nu=0$, e.g., see [15. It implies that the solutions of the ordinary differential equation $\frac{d \boldsymbol{x}}{d t}=\boldsymbol{u}(t, \boldsymbol{x})$ do never intersect and they remain smooth in $[0, T]$. Then, one obtains the argument

$$
\|\nabla \boldsymbol{u}\|_{L^{1}\left(0, T ; L^{\infty}(\Omega)\right)}
$$

in the exponential, e.g., compare [93, Rem. 7.40]. In this special case, the explicit dependency on inverse powers of the viscosity vanishes. Nevertheless, 
in all cases, the exponential factor becomes usually very large even for short times. But on the other hand, there is an abundance of practical simulations which shows that reliable flow simulations over long time intervals can be performed for flows whose scales in space and time can be resolved on a suitable mesh. In such situations, the errors are heavily overpredicted by the analytic bounds such that these bounds are useless.

- The derivation of quantitatively realistic error bounds for the Galerkin method applied to the discretization of flows whose scales in space and time can be resolved on a suitable mesh, possibly under some appropriate assumptions, is an open problem.

Note that examples can be constructed where the worst case, namely the exponential growth of errors with respect to time, can be observed, e.g., in [131, Ex. 5.2].

There are only few error bounds in the literature for approximations to the time-dependent Navier-Stokes equations where the constants are independent of explicit inverse powers of $\nu$ (apart from the dependency on $\nu^{-1}$ through norms of the exact solution), sometimes called semi-robust, uniform, or quasi-uniform estimates. Such bounds were derived by applying stabilization techniques that are also used for convection-diffusion equations, like the CIP method in 48 and the LPS method applied to the convective term in 12,58. On the other hand, semi-robust error bounds were obtained for discretizations with stabilization terms that were proposed for different purposes. Semi-robust error bounds of the Galerkin discretization with weakly divergence-free finite elements, even without additional stabilization term, can be found in [131. The analysis of the Galerkin method with grad-div stabilization, that allows the use of more general, not necessarily weakly divergence-free, finite elements can be found in [12,119 under the assumption that the grid is sufficiently fine $(h \lesssim \sqrt{\nu})$, in [58] with an assumption on the regularity of the finite element solution, and in 60] without any assumptions of this form. Grad-div stabilization was proposed for finite element discretizations to improve the approximation of the conservation of mass. In 60, optimal semi-robust bounds for the $L^{2}(\Omega)$ norm of the divergence of the velocity and the error of the pressure in the $L^{2}(\Omega)$ norm are derived. In [61, LPS stabilized methods are studied. Under the assumption that each component of the finite element velocity belongs to the pressure space, a semi-robust estimate is derived for a method with LPS stabilizations for the velocity gradient and the pressure gradient, and the convective term is bounded with the pressure gradient LPS stabilization.

- Finite element error analyses show that semi-robust estimates are obtained with stabilizations that were not proposed to stabilize dominant convection. The deeper reasons for this behavior are not yet understood.

An alternative way to derive semi-robust a priori and a posteriori error estimates was developed in 45 for the $2 \mathrm{~d}$ Navier-Stokes equations in vorticitystreamfunction formulation in the space-periodic setting. This approach is based on a scale separation of the velocity field together with a uniform bound- 
edness assumption for the large scales. However, already in [45] a number of reasons are given why this approach cannot be extended to the 3 d situation.

Standard finite element error analysis of the time-dependent Navier-Stokes equations derives error bounds for a sum of the velocity error in $L^{2}(\Omega)$ at the final time and a time-space energy error. Considering a family of shape-regular meshes, a conforming velocity finite element space that includes all piecewise polynomials of degree $k$, an appropriate pressure finite element space, and a sufficiently smooth exact solution, then the proved order of convergence is $k$. However, for the $L^{2}(\Omega)$ error at the final time, one might expect order $k+1$, which is also supported by numerical studies, e.g., see [93, Ex. 7.41]. A higher order than $k$ was proved for a CIP stabilized method in [48], namely

$$
\left\|\boldsymbol{u}-\boldsymbol{u}^{h}\right\|_{L^{\infty}\left(0, T ; L^{2}(\Omega)\right)} \leq C h^{k+1 / 2},
$$

where $C$ is independent of explicit negative powers of $\nu$ and it depends on $\|\boldsymbol{u}\|_{H^{k+1}(\Omega)}$ and $\|p\|_{H^{k+1}(\Omega)}$. Thus, half an order has been achieved with increasing the needed regularity for the pressure from $H^{k}(\Omega)$ to $H^{k+1}(\Omega)$. In 61, error bounds of type (16) are obtained for a LPS method with non inf-sup stable elements that adds a control of the fluctuation of the gradient and an LPS term stabilizing the pressure. As in [48, the pressure is assumed to be in $H^{k+1}(\Omega)$. An estimate of order $k+1$, which is however not semi-robust, is given in 71 for an IGA method.

- It is open whether optimal and semi-robust $L^{\infty}\left(0, T ; L^{2}(\Omega)^{d}\right)$ error bounds for the velocity can be proved for some method.

Pressure-robust discretizations for the Stokes equations, which are not weakly divergence-free, need only a modification of the discrete right-hand side, compare Section 3.1. However, the development of pressure-robust schemes for the incompressible Navier-Stokes equations requires additional modifications of the discretization of the temporal derivative and of the discrete nonlinear convective term, thus leading also to modifications of the matrix. There are already proposals on how to perform such modifications in practice, e.g., in [37, 117]. Finite element analysis can be found so far for the steady-state Navier-Stokes equations and the rotational form of the convective term 12 in [117.

- The finite element analysis of not weakly divergence-free pressure-robust discretizations for the time-dependent Navier-Stokes equations has still to be developed.

Since the behavior of turbulent flows is in some sense chaotic, the computation of a certain local quantity in time and space is usually not meaningful in applications. Hence, for turbulent flows, one is usually interested in temporal or spatio-temporal averages of quantities. So far, there are only very initial attempts to derive error estimates for such averaged quantities. In [97, few results for time-averaged errors in standard norms are presented. An a posteriori error estimator and an adaptive algorithm for time-averaged functionals of 
interest for (flow) problems with periodic or quasi-periodic behavior are proposed in [34. In [47, an error estimate for a time-averaged pressure computed with a CIP stabilization of the transient Oseen equations is derived.

- The numerical analysis for temporal or spatio-temporal averages of errors and quantities of interest has to be developed further.

As already mentioned in Section 3.2 in the last decade, one-grid preconditioners for linear algebraic saddle point problems arising in the discretization and linearization of the time-dependent Navier-Stokes equations have been developed that work efficiently, at least on serial or small parallel computers, e.g., in $[28,29,68,69]$.

In many applications, the flow domain depends on time, e.g., if problems with free surfaces are considered.

- Finite element analysis for the Navier-Stokes equations in time-dependent domains does not seem to be available.

\section{Summary}

Finite elements for scalar convection-dominated equations and incompressible flow problems - a never ending story? We hope that this paper provided the evidence that the end is not yet reached. In contrast, there are still many important open problems. Most of them seem to be quite challenging. To work at their solution requires a profound knowledge of the fields that are addressed in this paper. And even then, quick solutions, which are accompanied with fast publications, are rather unlikely.

We hope that this paper stimulates further research on finite element methods for convection-dominated problems and incompressible flow problems, in particular to solve the stated open problems.

Acknowledgments. The work of P. Knobloch was supported through the grant No. 16-03230S of the Czech Science Foundation. The work of J. Novo was supported by Spanish MINECO under grants MTM2013-42538-P (MINECO, ES) and MTM2016-78995-P (AEI/FEDER, UE).

We would like to thank an anonymous referee whose suggestions helped us to improve this paper.

\section{References}

1. Gabriel Acosta and Ricardo G. Durán. The maximum angle condition for mixed and nonconforming elements: application to the Stokes equations. SIAM J. Numer. Anal., 37(1):18-36, 1999.

2. Naveed Ahmed, Clemens Bartsch, Volker John, and Ulrich Wilbrandt. An assessment of solvers for saddle point problems emerging from the incompressible Navier-Stokes equations. Preprint 2408, WIAS Berlin, 2017. 
3. Mark Ainsworth, Gabriel R. Barrenechea, and Andreas Wachtel. Stabilization of high aspect ratio mixed finite elements for incompressible flow. SIAM J. Numer. Anal., 53(2):1107-1120, 2015.

4. Mark Ainsworth and Patrick Coggins. The stability of mixed $h p$-finite element methods for Stokes flow on high aspect ratio elements. SIAM J. Numer. Anal., 38(5):1721-1761, 2000 .

5. Alejandro Allendes, Francisco Durán, and Richard Rankin. Error estimation for loworder adaptive finite element approximations for fluid flow problems. IMA J. Numer. Anal., 36(4):1715-1747, 2016.

6. Thomas Apel, Tobias Knopp, and Gert Lube. Stabilized finite element methods with anisotropic mesh refinement for the Oseen problem. Appl. Numer. Math., 58(12):1830 1843,2008

7. Thomas Apel and H. Maharavo Randrianarivony. Stability of discretizations of the Stokes problem on anisotropic meshes. Math. Comput. Simulation, 61(3-6):437-447, 2003.

8. Thomas Apel and Gunar Matthies. Nonconforming, anisotropic, rectangular finite elements of arbitrary order for the Stokes problem. SIAM J. Numer. Anal., 46(4):1867$1891,2008$.

9. Thomas Apel and Serge Nicaise. The inf-sup condition for low order elements on anisotropic meshes. Calcolo, 41(2):89-113, 2004.

10. Thomas Apel, Serge Nicaise, and Joachim Schöberl. A non-conforming finite element method with anisotropic mesh grading for the Stokes problem in domains with edges. IMA J. Numer. Anal., 21(4):843-856, 2001.

11. Paul Arminjon and Alain Dervieux. Construction of TVD-like artificial viscosities on two-dimensional arbitrary FEM grids. J. Comput. Phys., 106(1):176-198, 1993.

12. Daniel Arndt, Helene Dallmann, and Gert Lube. Local projection FEM stabilization for the time-dependent incompressible Navier-Stokes problem. Numer. Methods Partial Differential Equations, 31(4):1224-1250, 2015.

13. Matthias Augustin, Alfonso Caiazzo, André Fiebach, Jürgen Fuhrmann, Volker John, Alexander Linke, and Rudolf Umla. An assessment of discretizations for convectiondominated convection-diffusion equations. Comput. Methods Appl. Mech. Engrg., 200(47-48):3395-3409, 2011.

14. Ivo Babuška. Error-bounds for finite element method. Numer. Math., 16:322-333, 1971.

15. Claude W. Bardos and Edriss S. Titi. Mathematics and turbulence: where do we stand? J. Turbul., 14(3):42-76, 2013.

16. Gabriel R. Barrenechea, Volker John, and Petr Knobloch. A local projection stabilization finite element method with nonlinear crosswind diffusion for convection-diffusionreaction equations. ESAIM Math. Model. Numer. Anal., 47(5):1335-1366, 2013.

17. Gabriel R. Barrenechea, Volker John, and Petr Knobloch. Some analytical results for an algebraic flux correction scheme for a steady convection-diffusion equation in one dimension. IMA J. Numer. Anal., 35(4):1729-1756, 2015.

18. Gabriel R. Barrenechea, Volker John, and Petr Knobloch. Analysis of algebraic flux correction schemes. SIAM J. Numer. Anal., 54(4):2427-2451, 2016.

19. Gabriel R. Barrenechea, Volker John, and Petr Knobloch. An algebraic flux correction scheme satisfying the discrete maximum principle and linearity preservation on general meshes. Math. Models Methods Appl. Sci., 27(3):525-548, 2017.

20. Gabriel R. Barrenechea and Frédéric Valentin. Consistent local projection stabilized finite element methods. SIAM J. Numer. Anal., 48(5):1801-1825, 2010.

21. Gabriel R. Barrenechea and Frédéric Valentin. A residual local projection method for the Oseen equation. Comput. Methods Appl. Mech. Engrg., 199(29-32):1906-1921, 2010 .

22. Gabriel R. Barrenechea and Frédéric Valentin. Beyond pressure stabilization: a loworder local projection method for the Oseen equation. Internat. J. Numer. Methods Engrg., 86(7):801-815, 2011.

23. Tomás P. Barrios, J. Manuel Cascón, and María González. Augmented mixed finite element method for the Oseen problem: a priori and a posteriori error analyses. Comput. Methods Appl. Mech. Engrg., 313:216-238, 2017. 
24. Y. Bazilevs, L. Beirão da Veiga, J. A. Cottrell, T. J. R. Hughes, and G. Sangalli. Isogeometric analysis: approximation, stability and error estimates for $h$-refined meshes. Math. Models Methods Appl. Sci., 16(7):1031-1090, 2006.

25. Y. Bazilevs, V. M. Calo, T. E. Tezduyar, and T. J. R. Hughes. $Y Z \beta$ discontinuity capturing for advection-dominated processes with application to arterial drug delivery. Internat. J. Numer. Methods Fluids, 54(6-8):593-608, 2007.

26. Roland Becker and Malte Braack. A finite element pressure gradient stabilization for the Stokes equations based on local projections. Calcolo, 38(4):173-199, 2001.

27. Roland Becker and Malte Braack. A two-level stabilization scheme for the NavierStokes equations. In M. Feistauer, V. Dolejší, P. Knobloch, and K. Najzar, editors, Numerical mathematics and advanced applications, pages 123-130. Springer, Berlin, 2004.

28. Michele Benzi and Maxim A. Olshanskii. An augmented Lagrangian-based approach to the Oseen problem. SIAM J. Sci. Comput., 28(6):2095-2113, 2006

29. Michele Benzi and Zhen Wang. Analysis of augmented Lagrangian-based preconditioners for the steady incompressible Navier-Stokes equations. SIAM J. Sci. Comput., 33(5):2761-2784, 2011.

30. Stefano Berrone. Robustness in a posteriori error analysis for FEM flow models. $\mathrm{Nu}$ mer. Math., 91(3):389-422, 2002.

31. Pavel Bochev and Max Gunzburger. An absolutely stable pressure-Poisson stabilized finite element method for the Stokes equations. SIAM J. Numer. Anal., 42(3):11891207, 2004.

32. Jay P. Boris and David L. Book. Flux-corrected transport. I. SHASTA, a fluid transport algorithm that works. J. Comput. Phys., 11(1):38-69, 1973.

33. Malte Braack. A stabilized finite element scheme for the Navier-Stokes equations on quadrilateral anisotropic meshes. M2AN Math. Model. Numer. Anal., 42(6):903-924 2008 .

34. Malte Braack, Erik Burman, and Nico Taschenberger. Duality based a posteriori error estimation for quasi-periodic solutions using time averages. SIAM J. Sci. Comput., 33(5):2199-2216, 2011.

35. Malte Braack, Gert Lube, and Lars Röhe. Divergence preserving interpolation on anisotropic quadrilateral meshes. Comput. Methods Appl. Math., 12(2):123-138, 2012.

36. Malte Braack and Piotr Boguslaw Mucha. Directional do-nothing condition for the Navier-Stokes equations. J. Comput. Math., 32(5):507-521, 2014.

37. Christian Brennecke, Alexander Linke, Christian Merdon, and Joachim Schöberl. Optimal and pressure-independent $L^{2}$ velocity error estimates for a modified CrouzeixRaviart Stokes element with BDM reconstructions. J. Comput. Math., 33(2):191-208, 2015 .

38. Franco Brezzi. On the existence, uniqueness and approximation of saddle-point problems arising from Lagrangian multipliers. Rev. Française Automat. Informat. Recherche Opérationnelle Sér. Rouge, 8(R-2):129-151, 1974.

39. Franco Brezzi and Michel Fortin. A minimal stabilisation procedure for mixed finite element methods. Numer. Math., 89(3):457-491, 2001.

40. Franco Brezzi and Juhani Pitkäranta. On the stabilization of finite element approximations of the Stokes equations. In Efficient solutions of elliptic systems (Kiel, 1984), volume 10 of Notes Numer. Fluid Mech., pages 11-19. Friedr. Vieweg, Braunschweig, 1984

41. Alexander N. Brooks and Thomas J. R. Hughes. Streamline upwind/Petrov-Galerkin formulations for convection dominated flows with particular emphasis on the incompressible Navier-Stokes equations. Comput. Methods Appl. Mech. Engrg., 32(1-3):199259, 1982.

42. A. Buffa, C. de Falco, and G. Sangalli. IsoGeometric Analysis: stable elements for the 2D Stokes equation. Internat. J. Numer. Methods Fluids, 65(11-12):1407-1422, 2011.

43. Jannis Bulling, Volker John, and Petr Knobloch. Isogeometric analysis for flows around a cylinder. Appl. Math. Lett., 63:65-70, 2017.

44. Erik Burman. A posteriori error estimation for interior penalty finite element approximations of the advection-reaction equation. SIAM J. Numer. Anal., 47(5):3584-3607, 2009. 
45. Erik Burman. Robust error estimates for stabilized finite element approximations of the two dimensional Navier-Stokes' equations at high Reynolds number. Comput. Methods Appl. Mech. Engrg., 288:2-23, 2015.

46. Erik Burman and Alexandre Ern. Stabilized Galerkin approximation of convectiondiffusion-reaction equations: discrete maximum principle and convergence. Math. Comp., 74(252):1637-1652 (electronic), 2005.

47. Erik Burman, Alexandre Ern, and Miguel A. Fernández. Fractional-step methods and finite elements with symmetric stabilization for the transient Oseen problem. ESAIM: M2AN, 51(2):487-507, 2017.

48. Erik Burman and Miguel A. Fernández. Continuous interior penalty finite element method for the time-dependent Navier-Stokes equations: space discretization and convergence. Numer. Math., 107(1):39-77, 2007.

49. Erik Burman, Johnny Guzmán, and Dmitriy Leykekhman. Weighted error estimates of the continuous interior penalty method for singularly perturbed problems. IMA J. Numer. Anal., 29(2):284-314, 2009.

50. Erik Burman and Peter Hansbo. Edge stabilization for Galerkin approximations of convection-diffusion-reaction problems. Comput. Methods Appl. Mech. Engrg., 193(1516):1437-1453, 2004.

51. Erik Burman and Peter Hansbo. Edge stabilization for the generalized Stokes problem: a continuous interior penalty method. Comput. Methods Appl. Mech. Engrg., 195(19$22): 2393-2410,2006$.

52. Erik Burman and Isaac P. Santos. Error estimates for transport problems with high Péclet number using a continuous dependence assumption. J. Comput. Appl. Math., 309:267-286, 2017.

53. Sergey Charnyi, Timo Heister, Maxim A. Olshanskii, and Leo G. Rebholz. On conservation laws of Navier-Stokes Galerkin discretizations. Journal of Computational Physics, 337:289 - 308, 2017.

54. Hongsen Chen. Pointwise error estimates for finite element solutions of the Stokes problem. SIAM J. Numer. Anal., 44(1):1-28, 2006.

55. Evgenii V. Chizhonkov and Maxim A. Olshanskii. On the domain geometry dependence of the LBB condition. M2AN Math. Model. Numer. Anal., 34(5):935-951, 2000.

56. Ramon Codina and Jordi Blasco. A finite element formulation for the Stokes problem allowing equal velocity-pressure interpolation. Comput. Methods Appl. Mech. Engrg., 143(3-4):373-391, 1997.

57. Michel Crouzeix and Pierre-Arnaud Raviart. Conforming and nonconforming finite element methods for solving the stationary Stokes equations. I. Rev. Française Automat. Informat. Recherche Opérationnelle Sér. Rouge, 7(R-3):33-75, 1973.

58. Helene Dallmann and Daniel Arndt. Stabilized finite element methods for the Oberbeck-Boussinesq model. J. Sci. Comput., 69(1):244-273, 2016.

59. Javier de Frutos, Bosco García-Archilla, Volker John, and Julia Novo. An adaptive SUPG method for evolutionary convection-diffusion equations. Comput. Methods Appl. Mech. Engrg., 273:219-237, 2014.

60. Javier de Frutos, Bosco García-Archilla, Volker John, and Julia Novo. Analysis of the grad-div stabilization for the time-dependent Navier-Stokes equations with inf-sup stable finite elements. Adv. Comput. Math., 2017. In press.

61. Javier de Frutos, Bosco García-Archilla, Volker John, and Julia Novo. Semi-robust local projection stabilization for non inf-sup stable discretizations of the evolutionary Navier-Stokes equations. Technical Report 1709.01011, arXiv, 2017.

62. Javier de Frutos, Bosco García-Archilla, and Julia Novo. Local error estimates for the SUPG method applied to evolutionary convection-reaction-diffusion equations. J. Sci. Comput., 66(2):528-554, 2016.

63. Clark R. Dohrmann and Pavel B. Bochev. A stabilized finite element method for the Stokes problem based on polynomial pressure projections. Internat. J. Numer. Methods Fluids, 46(2):183-201, 2004.

64. Jim Douglas, Jr. and Jun Ping Wang. An absolutely stabilized finite element method for the Stokes problem. Math. Comp., 52(186):495-508, 1989.

65. Shaohong Du and Zhimin Zhang. A robust residual-type a posteriori error estimator for convection-diffusion equations. J. Sci. Comput., 65(1):138-170, 2015. 
66. Francisco Durango and Julia Novo. Two-grid mixed finite-element approximations to the Navier-Stokes equations based on a Newton type-step. J. Sci. Comput., 2017. In press.

67. Martin Eigel and Christian Merdon. Equilibration a posteriori error estimation for convection-diffusion-reaction problems. J. Sci. Comput., 67(2):747-768, 2016.

68. Howard Elman, Victoria E. Howle, John Shadid, Robert Shuttleworth, and Ray Tuminaro. Block preconditioners based on approximate commutators. SIAM J. Sci. Comput., 27(5):1651-1668, 2006.

69. Howard C. Elman, David J. Silvester, and Andrew J. Wathen. Finite elements and fast iterative solvers: with applications in incompressible fluid dynamics. Numerical Mathematics and Scientific Computation. Oxford University Press, Oxford, second edition, 2014.

70. John A. Evans and Thomas J. R. Hughes. Isogeometric divergence-conforming Bsplines for the steady Navier-Stokes equations. Math. Models Methods Appl. Sci., 23(8):1421-1478, 2013.

71. John A. Evans and Thomas J.R. Hughes. Isogeometric divergence-conforming B-splines for the unsteady Navier-Stokes equations. J. Comp. Phys., 241:141 - 167, 2013.

72. Richard S. Falk and Michael Neilan. Stokes complexes and the construction of stable finite elements with pointwise mass conservation. SIAM J. Numer. Anal., 51(2):13081326, 2013.

73. Vivette Girault, Ricardo H. Nochetto, and Larkin Ridgway Scott. Max-norm estimates for Stokes and Navier-Stokes approximations in convex polyhedra. Numer. Math., 131(4):771-822, 2015.

74. Vivette Girault and Pierre-Arnaud Raviart. Finite element approximation of the Navier-Stokes equations, volume 749 of Lecture Notes in Mathematics. SpringerVerlag, Berlin-New York, 1979.

75. Vivette Girault and Pierre-Arnaud Raviart. Finite element methods for Navier-Stokes equations. Theory and algorithms, volume 5 of Springer Series in Computational Mathematics. Springer-Verlag, Berlin, 1986.

76. Vivette Girault and Larkin Ridgway Scott. A quasi-local interpolation operator preserving the discrete divergence. Calcolo, 40(1):1-19, 2003.

77. Roland Glowinski. Finite element methods for incompressible viscous flow. In Handbook of numerical analysis, Vol. IX, Handb. Numer. Anal., IX, pages 3-1176. NorthHolland, Amsterdam, 2003

78. Sergei Konstantinovich Godunov. A difference method for numerical calculation of discontinuous solutions of the equations of hydrodynamics. Mat. Sb. (N.S.), 47 (89):271306, 1959.

79. Johnny Guzmán and Dmitriy Leykekhman. Pointwise error estimates of finite element approximations to the Stokes problem on convex polyhedra. Math. Comp., 81(280):1879-1902, 2012.

80. Johnny Guzmán and Michael Neilan. Conforming and divergence-free Stokes elements on general triangular meshes. Math. Comp., 83(285):15-36, 2014.

81. Johnny Guzmán and Manuel A. Sánchez. Max-norm stability of low order Taylor-Hood elements in three dimensions. J. Sci. Comput., 65(2):598-621, 2015.

82. Guillermo Hauke, Mohamed H. Doweidar, and Daniel Fuster. A posteriori error estimation for computational fluid dynamics: the variational multiscale approach. In Multiscale methods in computational mechanics, volume 55 of Lect. Notes Appl. Comput. Mech., pages 19-38. Springer, Dordrecht, 2011.

83. Guillermo Hauke, Mohamed H. Doweidar, Daniel Fuster, Antonio Gómez, and Javier Sayas. Application of variational a-posteriori multiscale error estimation to higherorder elements. Comput. Mech., 38(4-5):356-389, 2006.

84. Guillermo Hauke, Daniel Fuster, and Mohamed H. Doweidar. Variational multiscale aposteriori error estimation for multi-dimensional transport problems. Comput. Methods Appl. Mech. Engrg., 197(33-40):2701-2718, 2008.

85. Babak S. Hosseini, Matthias Möller, and Stefan Turek. Isogeometric analysis of the Navier-Stokes equations with Taylor-Hood B-spline elements. Appl. Math. Comput., 267:264-281, 2015. 
86. T. J. R. Hughes, J. A. Cottrell, and Y. Bazilevs. Isogeometric analysis: CAD, finite elements, NURBS, exact geometry and mesh refinement. Comput. Methods Appl. Mech. Engrg., 194(39-41):4135-4195, 2005.

87. Thomas J. R. Hughes. Multiscale phenomena: Green's functions, the Dirichlet-toNeumann formulation, subgrid scale models, bubbles and the origins of stabilized methods. Comput. Methods Appl. Mech. Engrg., 127(1-4):387-401, 1995.

88. Thomas J. R. Hughes and Alexander Brooks. A multidimensional upwind scheme with no crosswind diffusion. In Finite element methods for convection dominated flows (Papers, Winter Ann. Meeting Amer. Soc. Mech. Engrs., New York, 1979), volume 34 of $A M D$, pages 19-35. Amer. Soc. Mech. Engrs. (ASME), New York, 1979

89. Thomas J. R. Hughes and Leopoldo P. Franca. A new finite element formulation for computational fluid dynamics. VII. The Stokes problem with various well-posed boundary conditions: symmetric formulations that converge for all velocity/pressure spaces. Comput. Methods Appl. Mech. Engrg., 65(1):85-96, 1987.

90. Thomas J. R. Hughes, Leopoldo P. Franca, and Marc Balestra. A new finite element formulation for computational fluid dynamics. V. Circumventing the Babuška-Brezzi condition: a stable Petrov-Galerkin formulation of the Stokes problem accommodating equal-order interpolations. Comput. Methods Appl. Mech. Engrg., 59(1):85-99, 1986.

91. Thomas J. R. Hughes and Giancarlo Sangalli. Variational multiscale analysis: the finescale Green's function, projection, optimization, localization, and stabilized methods. SIAM J. Numer. Anal., 45(2):539-557, 2007.

92. Volker John. A numerical study of a posteriori error estimators for convection-diffusion equations. Comput. Methods Appl. Mech. Engrg., 190(5-7):757-781, 2000.

93. Volker John. Finite element methods for incompressible flow problems, volume 51 of Springer Series in Computational Mathematics. Springer, Cham, 2016.

94. Volker John, Kristine Kaiser, and Julia Novo. Finite element methods for the incompressible Stokes equations with variable viscosity. ZAMM Z. Angew. Math. Mech., 96(2):205-216, 2016.

95. Volker John and Petr Knobloch. On spurious oscillations at layers diminishing (SOLD) methods for convection-diffusion equations. I. A review. Comput. Methods Appl. Mech. Engrg., 196(17-20):2197-2215, 2007.

96. Volker John and Petr Knobloch. On spurious oscillations at layers diminishing (SOLD) methods for convection-diffusion equations. II. Analysis for $P_{1}$ and $Q_{1}$ finite elements. Comput. Methods Appl. Mech. Engrg., 197(21-24):1997-2014, 2008.

97. Volker John, William Layton, and Carolina Cardoso Manica. Convergence of timeaveraged statistics of finite element approximations of the Navier-Stokes equations. SIAM J. Numer. Anal., 46(1):151-179, 2007.

98. Volker John, Alexander Linke, Christian Merdon, Michael Neilan, and Leo G. Rebholz. On the divergence constraint in mixed finite element methods for incompressible flows. SIAM Rev., 59:492-544, 2017.

99. Volker John, Teodora Mitkova, Michael Roland, Kai Sundmacher, Lutz Tobiska, and Andreas Voigt. Simulations of population balance systems with one internal coordinate using finite element methods. Chemical Engineering Science, 64(4):733 - 741, 2009.

100. Volker John and Julia Novo. On (essentially) non-oscillatory discretizations of evolutionary convection-diffusion equations. J. Comput. Phys., 231(4):1570-1586, 2012.

101. Volker John and Julia Novo. A robust SUPG norm a posteriori error estimator for stationary convection-diffusion equations. Comput. Methods Appl. Mech. Engrg., 255:289-305, 2013.

102. Volker John and Ellen Schmeyer. Finite element methods for time-dependent convection-diffusion-reaction equations with small diffusion. Comput. Methods Appl. Mech. Engrg., 198(3-4):475-494, 2008.

103. Volker John and Liesel Schumacher. A study of isogeometric analysis for scalar convection-diffusion equations. Appl. Math. Lett., 27:43-48, 2014.

104. Claes Johnson, Alfred H. Schatz, and Lars B. Wahlbin. Crosswind smear and pointwise errors in streamline diffusion finite element methods. Math. Comp., 49(179):25-38, 1987.

105. Petr Knobloch. Improvements of the Mizukami-Hughes method for convectiondiffusion equations. Comput. Methods Appl. Mech. Engrg., 196(1-3):579-594, 2006. 
106. T. Knopp, G. Lube, and G. Rapin. Stabilized finite element methods with shock capturing for advection-diffusion problems. Comput. Methods Appl. Mech. Engrg., 191(27-28):2997-3013, 2002.

107. Dmitri Kuzmin. On the design of general-purpose flux limiters for finite element schemes. I. Scalar convection. J. Comput. Phys., 219(2):513-531, 2006.

108. Dmitri Kuzmin. Algebraic flux correction for finite element discretizations of coupled systems. In Manolis Papadrakakis, Eugenio Oñate, and Bernard Schrefler, editors, Proceedings of the Int. Conf. on Computational Methods for Coupled Problems in Science and Engineering, pages 1-5. CIMNE, Barcelona, 2007.

109. Dmitri Kuzmin. Linearity-preserving flux correction and convergence acceleration for constrained Galerkin schemes. J. Comput. Appl. Math., 236(9):2317-2337, 2012.

110. Dmitri Kuzmin and Matthias Möller. Algebraic flux correction I. Scalar conservation laws. In Dmitri Kuzmin, Rainald Löhner, and Stefan Turek, editors, Flux-Corrected Transport. Principles, Algorithms, and Applications, pages 155-206. Springer-Verlag, Berlin, 2005.

111. Dmitri Kuzmin and Stefan Turek. High-resolution FEM-TVD schemes based on a fully multidimensional flux limiter. J. Comput. Phys., 198(1):131-158, 2004.

112. William Layton. Introduction to the numerical analysis of incompressible viscous flows, volume 6 of Computational Science 8 Engineering. Society for Industrial and Applied Mathematics (SIAM), Philadelphia, PA, 2008.

113. Philip L. Lederer, Alexander Linke, Christian Merdon, and Joachim Schöberl. Divergence-free reconstruction operators for pressure-robust Stokes discretizations with continuous pressure finite elements. SIAM J. Numer. Anal., 55(3):1291-1314, 2017.

114. Qifeng Liao and David Silvester. Robust stabilized Stokes approximation methods for highly stretched grids. IMA J. Numer. Anal., 33(2):413-431, 2013.

115. Alexander Linke. On the role of the Helmholtz decomposition in mixed methods for incompressible flows and a new variational crime. Comput. Methods Appl. Mech. Engrg., 268:782-800, 2014.

116. Alexander Linke, Gunar Matthies, and Lutz Tobiska. Robust arbitrary order mixed finite element methods for the incompressible Stokes equations with pressure independent velocity errors. ESAIM Math. Model. Numer. Anal., 50(1):289-309, 2016.

117. Alexander Linke and Christian Merdon. Pressure-robustness and discrete Helmholtz projectors in mixed finite element methods for the incompressible Navier-Stokes equations. Comput. Methods Appl. Mech. Engrg., 311:304-326, 2016.

118. Rainald Löhner, Ken Morgan, Jaime Peraire, and Mehdi Vahdati. Finite element fluxcorrected transport (FEM-FCT) for the Euler and Navier-Stokes equations. Int. J. Numer. Methods Fluids, 7(10):1093-1109, 1987.

119. Gert Lube, Daniel Arndt, and Helene Dallmann. Understanding the limits of inf-sup stable Galerkin-FEM for incompressible flows. In Boundary and interior layers, computational and asymptotic methods-BAIL 2014, volume 108 of Lect. Notes Comput. Sci. Eng., pages 147-169. Springer, Cham, 2015.

120. Gert Lube and Gerd Rapin. Residual-based stabilized higher-order FEM for advectiondominated problems. Comput. Methods Appl. Mech. Engrg., 195(33-36):4124-4138, 2006 .

121. Stefano Micheletti, Simona Perotto, and Marco Picasso. Stabilized finite elements on anisotropic meshes: a priori error estimates for the advection-diffusion and the Stokes problems. SIAM J. Numer. Anal., 41(3):1131-1162, 2003.

122. Akira Mizukami and Thomas J. R. Hughes. A Petrov-Galerkin finite element method for convection-dominated flows: an accurate upwinding technique for satisfying the maximum principle. Comput. Methods Appl. Mech. Engrg., 50(2):181-193, 1985.

123. Uno Nävert. A Finite Element Method for Convection-Diffusion Problems. $\mathrm{PhD}$ thesis, Chalmers University of Technology, 1982.

124. Koichi Niijima. Pointwise error estimates for a streamline diffusion finite element scheme. Numer. Math., 56(7):707-719, 1990.

125. Hans-Görg Roos and Martin Stynes. Some open questions in the numerical analysis of singularly perturbed differential equations. Comput. Methods Appl. Math., 15(4):531550, 2015. 
126. Hans-Görg Roos, Martin Stynes, and Lutz Tobiska. Numerical methods for singularly perturbed differential equations. Convection-diffusion and flow problems, volume 24 of Springer Series in Computational Mathematics. Springer-Verlag, Berlin, 1996.

127. Hans-Görg Roos, Martin Stynes, and Lutz Tobiska. Robust numerical methods for singularly perturbed differential equations. Convection-diffusion-reaction and flow problems, volume 24 of Springer Series in Computational Mathematics. Springer-Verlag, Berlin, second edition, 2008

128. Youcef Saad. A flexible inner-outer preconditioned GMRES algorithm. SIAM J. Sci. Comput., 14(2):461-469, 1993.

129. Giancarlo Sangalli. Robust a-posteriori estimator for advection-diffusion-reaction problems. Math. Comp., 77(261):41-70 (electronic), 2008.

130. Dominik Schötzau, Christoph Schwab, and Rolf Stenberg. Mixed $h p$-FEM on anisotropic meshes. II. Hanging nodes and tensor products of boundary layer meshes. Numer. Math., 83(4):667-697, 1999.

131. Philipp W. Schroeder and Gert Lube. Pressure-robust analysis of divergence-free and conforming FEM for evolutionary incompressible Navier-Stokes flows. J. Num. Math., 2017. Accepted for publication.

132. Kristina Schwegler and Markus Bause. Goal-oriented a posteriori error control for nonstationary convection-dominated transport problems. Technical Report 1601.06544 arXiv, 2016.

133. Larkin Ridgway Scott and Michael Vogelius. Conforming finite element methods for incompressible and nearly incompressible continua. In Large-scale computations in fluid mechanics, Part 2 (La Jolla, Calif., 1983), volume 22 of Lectures in Appl. Math., pages 221-244. Amer. Math. Soc., Providence, RI, 1985.

134. Hendrik Speleers, Carla Manni, Francesca Pelosi, and M. Lucia Sampoli. Isogeometric analysis with Powell-Sabin splines for advection-diffusion-reaction problems. Comput. Methods Appl. Mech. Engrg., 221/222:132-148, 2012.

135. Masahisa Tabata and Daisuke Tagami. Error estimates for finite element approximations of drag and lift in nonstationary Navier-Stokes flows. Japan J. Indust. Appl. Math., 17(3):371-389, 2000.

136. Lutz Tobiska and Rüdiger Verfürth. Robust a posteriori error estimates for stabilized finite element methods. IMA J. Numer. Anal., 35(4):1652-1671, 2015.

137. S. Pratap Vanka. Block-implicit multigrid solution of Navier-Stokes equations in primitive variables. J. Comput. Phys., 65(1):138-158, 1986.

138. Rüdiger Verfürth. A posteriori error estimators for convection-diffusion equations. Numer. Math., 80(4):641-663, 1998.

139. Rüdiger Verfürth. Robust a posteriori error estimates for stationary convectiondiffusion equations. SIAM J. Numer. Anal., 43(4):1766-1782 (electronic), 2005.

140. Ulrich Wilbrandt, Clemens Bartsch, Naveed Ahmed, Najib Alia, Felix Anker, Laura Blank, Alfonso Caiazzo, Sashikumaar Ganesan, Swetlana Giere, Gunar Matthies, Raviteja Meesala, Abdus Shamim, Jagannath Venkatesan, and Volker John. ParMooN-A modernized program package based on mapped finite elements. Comput. Math. Appl., 74(1):74-88, 2017.

141. Steven T. Zalesak. Fully multidimensional flux-corrected transport algorithms for fluids. J. Comput. Phys., 31(3):335-362, 1979.

142. Shangyou Zhang. A new family of stable mixed finite elements for the 3D Stokes equations. Math. Comp., 74(250):543-554, 2005.

143. Guo Hui Zhou and Rolf Rannacher. Pointwise superconvergence of the streamline diffusion finite-element method. Numer. Methods Partial Differential Equations, 12(1):123$145,1996$.

144. Guohui Zhou. How accurate is the streamline diffusion finite element method? Math. Comp., 66(217):31-44, 1997. 